\title{
Study on the fluid dynamics of nitrogen and hydrogen gases subjected to wires element in monolithic channel
}

\author{
Amirah Rashidi, ${ }^{1, *}$, Zamri Abdullah ${ }^{1}$, and Salman Kashif ${ }^{1}$ \\ ${ }^{1}$ Chemical Engineering Department, Universiti Teknologi PETRONAS, 32610 Bandar Seri Iskandar, Perak Darul Ridzuan, Malaysia
}

\begin{abstract}
Ammonia has a very significant value in the fertilizer industry where it was being synthesized via Haber-Bosch process in the early 19th century. As the process utilize high operating conditions, it imposes high capital cost and is an energy-consuming process. Due to this unsustainable process, researchers have initiated an alternative to overcome this drawback by performing a simulation in microfluidic environment using ambient temperature and pressure $\left(25^{\circ} \mathrm{C}\right.$ and $\left.1 \mathrm{~atm}\right)$. Wires element configured in a 50 $\mathrm{mm} \times 10 \mathrm{~mm}$, (L x D) dimension monolithic channel with different spacing and number of wires, arranged axially in $60^{\circ}$ pitch have been introduced to investigate the dynamic mixing of nitrogen and hydrogen for ammonia synthesis. As the wires are configured in a different manner, the results show dissimilar volume fraction profile, contours and mixing index. Creating suitable obstruction with larger obstruction space enhanced the mixing. Reducing spacing from $2 \mathrm{~mm}$ to $1.5 \mathrm{~mm}$ illustrates fluctuating velocity at the centre of the channel causing the flow velocity become less than the set velocity $0.05 \mathrm{~ms}^{-1}$. By substituting from 19 wires to 13 wires to the flow, chaotic advection occurs lead to the increased of mixing index up to $94 \%$.
\end{abstract}

Keywords. monolithic channel / computational fluid dynamic / dynamic mixing / ANSYS CFX / ammonia

\section{Introduction}

Ammonia $\left(\mathrm{NH}_{3}\right)$ is well-known in the industry for various applications with a high percentage, of $80 \%$ approximately from the total production, has been applied in synthetic fertilizer, therefore causing a great market demand in the industry business [1]. Consumption of fertilizer increased significantly in 2010 and is expected to grow in a stabilized way during the following years of the forecast period. World demand for total fertilizer is estimated to grow at $2.0 \%$ per annum from 2011 to 2015 [2].

Regrettably, the current production is capital and energy intensive but can only yield $10-20 \%$ of $\mathrm{NH}_{3}$ [3]. This process of producing $\mathrm{NH}_{3}$ through reaction of nitrogen $\left(\mathrm{N}_{2}\right)$ and hydrogen $\left(\mathrm{H}_{2}\right)$ which is known as Haber-Bosch process obliges to operate under a high operating condition temperature $\left(400-500^{\circ} \mathrm{C}\right)$ and pressure (150-300 bar) where such circumstances requires a very careful handling as long with increasing the hazards to the operators and the plant facilities.

A new promising method to attain the high yield of $\mathrm{NH}_{3}$ at ambient condition in microfluidic environment [4] is discovered by using wires element in monolithic channel. This method is seen as an alternative to improvise the efficiency of $\mathrm{NH}_{3}$ production by exposing the raw materials, $\mathrm{H}_{2}$ and $\mathrm{N}_{2}$ to high surface area to volume ratio which have been proven previously by having parallel and perpendicular obstruction to enhance the dynamic mixing. The parallel and perpendicular placement leads to a favorable condition where the mixing index attains approximately $90 \%$ with the desired ratio for the raw materials to react [5]. As compared to simple square pitched arrangement, the possibility of having wires placed at $60^{\circ}$ pitch has been explored in this work to elevate the yield of $\mathrm{NH}_{3}$.

The usage of smaller dimension channel is proven to be more effective to obtain great reaction yield and product selectivity [6]. This reactor enables chemical reactions to be performed in reaction space several orders of magnitude smaller than conventional batch reactor [7]. The downscaling of devices has brought attractive features to the channel such as equipment installation time and time taken for the reaction to reach steady state has been reduced. The hazards and process safety risks may be reduced since the quantity of substances used in the channel is much more less.

Mixing is hardly achieved in minute system without modification of geometry. Mixing plays a significant role to increase the conversion of $\mathrm{N}_{2}$ and $\mathrm{H}_{2}$ to form $\mathrm{NH}_{3}$. Mainly the importance is to reduce inhomogeneity that leads to secondary effects such as undesired reaction and change in properties [8]. Furthermore, great flow dynamic is preferred in a channel if intimate mixing is desired 
between two streams [9]. Several patterns of channel were investigated formerly to address the significant of mixing such as serpentine shaped channel [10], linked twist map barriers [11], floor-grooved channel [12] and rhombic micromixer [13]. In this project, the simulation is done through configuring the wires assembly in parallel arrangement inside the channel that will induce dynamic mixing via chaotic advection to increase the efficiency of ammonia production.

\section{Simulation methodologies}

Simulation is performed using ANSYS CFX software. Typical methodologies in CFD apply such as creation of geometry, development of mesh, and post-solver analysis as display in Figure 1. The project emphasizes on the 'synthesis of ammonia'. However, for the scope of this work, only flow dynamics without the reaction element will be simulated. It is important to create a geometry that will generate a great flow for the mixing.

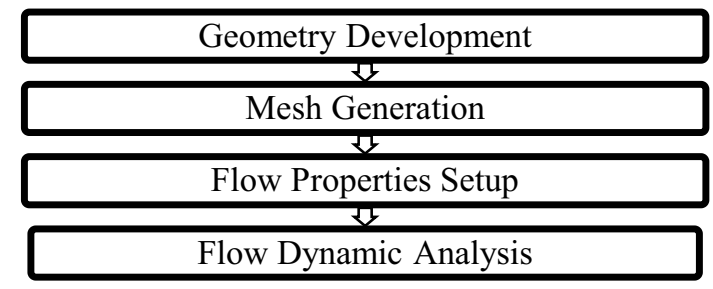

Fig. 1. Flow of simulation methodology.

\subsection{Geometry development}

As shown in Figure 2 the channel dimension is $10 \mathrm{~mm}$ (D) $x 50 \mathrm{~mm}(\mathrm{~L})$ while the diameters of the wires are $1 \mathrm{~mm}$. The wires assembly started at a distance on $3 \mathrm{~mm}$ from the inlet and ended $3 \mathrm{~mm}$ before the outlet as a mean to induce pre-mixing upon flowing inside which are constant for all three designs. The wires are arranged parallel to the channel with different spacing of wires and different number of wires aimed to create higher mixing efficiency.

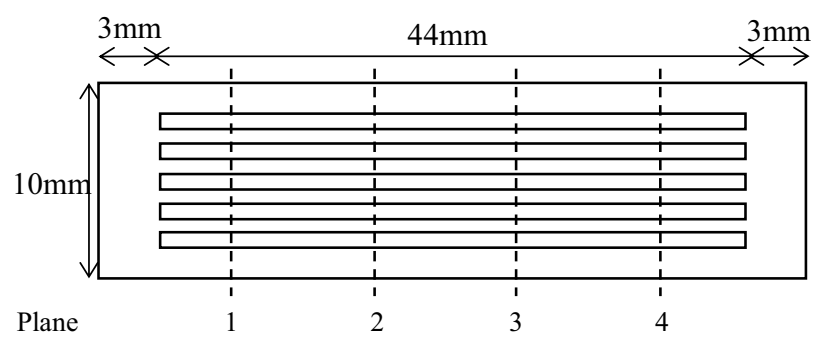

Fig. 2. General dimension of a monolithic channel.

Table 1 illustrates three designs that were developed in this project and the flow dynamics of the nitrogen and hydrogen gases throughout the monolithic channel will be observed and recorded. As it is arranged in parallel order, there is no intersection of wires and no change in wires path.
Table 1. Configuration of channel in radial view.

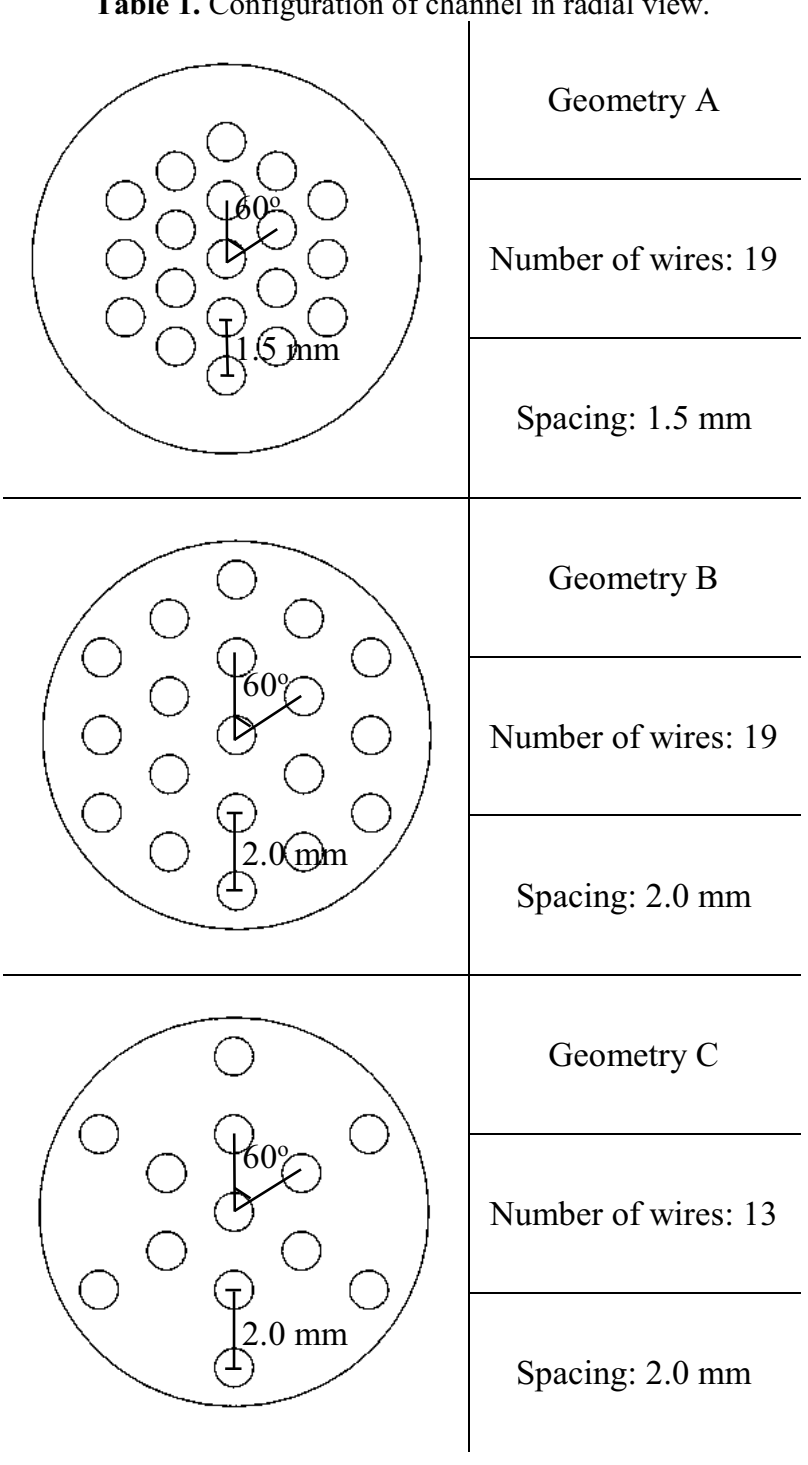

\subsection{Mesh Generation}

The mesh properties for geometry B were set up to fine, coarse and medium. Per the orthogonal factor, mixing index and time taken to run the program, coarse mesh was selected to be the relevance centre for all design since there was not much difference in mixing index curve making mesh finer as shown in Figure 3.

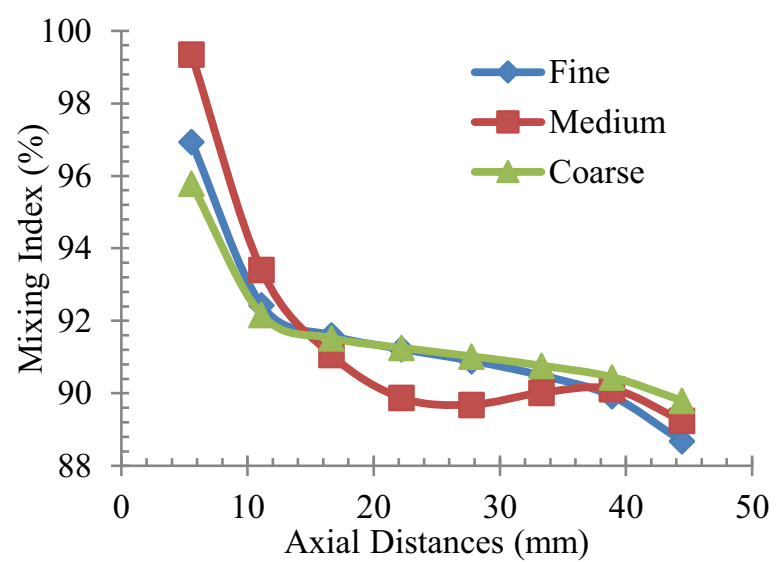

Fig. 3. Mixing index for different type of mesh.

\footnotetext{
${ }^{*}$ Corresponding author: nuramirahrashidi@gmail.com
} 


\subsection{Flow properties setup}

Once the geometry designs for the configurations and the meshing are accomplished, several pre-setup inputs are required by ANSYS before stimulating the design. The physics are listed in the Table 2.

Table 2. Flow parameters setup.

\begin{tabular}{cc}
\hline Parameter & Flow Properties \\
\hline Gas Inlet Velocity & $0.05 \mathrm{~ms}^{-1}$ \\
\hline Volume Fraction & $\mathrm{H}_{2}: \mathrm{N}_{2}=0.75: 0.25$ \\
\hline Temperature & $25^{\circ} \mathrm{C}$ \\
\hline Pressure & $1 \mathrm{~atm}$ \\
\hline Heat Transfer Model & Isothermal \\
\hline Turbulence Model & K-Epsilon \\
\hline Simulation Mode & Steady State
\end{tabular}

\subsection{Mixing index}

The main indicator to measure the proximity of the flow ratio of both gasses is by using mixing index. With the intention of quantifying mixing efficiency, volume fraction of hydrogen data from CFD simulation is extracted from each line that have been constructed in the monolithic channel. 8 samples that are taken along the channel length as shown in Figure 4 are used to calculate the mixing index.

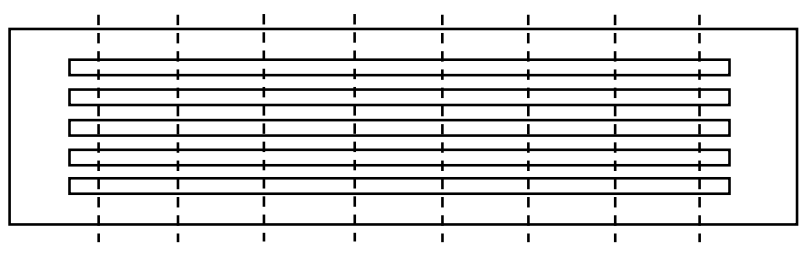

$\begin{array}{llllllll}5.6 & 11.1 & 16.7 & 22.2 & 27.8 & 33.3 & 38.9 & 44.4\end{array}$

Fig. 4. Distance in $\mathrm{mm}$ of sample taken along monolithic channel for mixing index calculation

Based on the points taken, mixing index graph will be created using equation 1 and 2 .

$$
\begin{gathered}
\gamma=\sqrt{\frac{1}{N} \sum\left(C_{i}-\overline{C_{m}}\right)^{2}} \\
M=1-\sqrt{\frac{\gamma^{2}}{\gamma_{\text {max }}^{2}}}
\end{gathered}
$$

\begin{tabular}{|ll}
\hline \multicolumn{2}{l}{ Nomenclature } \\
$\gamma$ & Variance \\
$N$ & Number of sampling point \\
$C_{i}$ & Mole fraction at point/node $i$ \\
$C_{m}$ & Optimal mixing fraction \\
$M$ & Mixing index \\
$\gamma^{2}$ & Actual variance \\
$\gamma_{\max }^{2}$ & Maximum variance from optimal mixing fraction
\end{tabular}

\section{Results and discussion}

The volume fraction and velocity of both nitrogen and hydrogen gases are extracted from the simulation in the form of contour plots in radial and axial manner. Each axial arrangement consists of four planes which are measured equally throughout the channel to observe the mixing of both gasses.

Mixing index for each geometry are calculated and presented in line chart to express which geometry create greater mixing to produce ammonia. The range of velocity profile is standardized from $0 \mathrm{~ms}^{-1}$ until $0.09 \mathrm{~ms}^{-1}$ while the range for volume fraction is from 0 to 1 which is illustrated at the legend on the right side of the tables. The results are taken from CFD Post.

Based on the results obtained from the simulation, effect of spacing between wires and effect of number of wires are investigated. Geometry A and B comprises the same geometry with different spacing of wires, $1.5 \mathrm{~mm}$ and $2.0 \mathrm{~mm}$. Geometry B and $\mathrm{C}$ are studied with different number of wires, 19 wires and 13 wires respectively.

\subsection{Effect of spacing of wires}

Increasing the parallel spacing between the wires lead to a significant effect on mixing index.

Rendering to the $\mathrm{XY}$-axis velocity contour of Figure 5 , nitrogen particles in Geometry B are having more stable velocity while Geometry A shows inconsistent velocity at the center of the channel as the arrangement of the wires is closely packed at that position providing less space for mixing which leads to unfavorable outcome.

Velocity contour of Geometry B presented in Figure 6 demonstrates a fast moving of hydrogen gas at $0.063 \mathrm{~ms}^{-}$ 1 to $0.072 \mathrm{~ms}^{-1}$ throughout the channel. The axial obstruction of Geometry A generates higher velocity of hydrogen at the inner wall of channel which can cause it hard for the comparatively heavier nitrogen gas to have the similar pace of the hydrogen gas in that region.

However, as shown in Figure 7, Geometry B is not able to produce consistent volume fraction of nitrogen and hydrogen ratio, 3:1 to enhance the collision. Geometry A possess volume fraction of nitrogen that is lower than 0.2 in the centre area at the inlet of the channel.

Referring to Figure 8, the centreline in YZ-axis of Geometry B is seen to have steady velocity of nitrogen while the hydrogen possesses higher velocity than the set velocity. For Geometry A, velocity of hydrogen gas is lower at the inlet part than the outlet of the channel.

As the surface area for mixing of raw materials of Geometry B is higher, the mixing index for this design elevated better than Geometry A as displayed in Figure 13 [10].

Therefore, Geometry $\mathrm{C}$ is created using $2 \mathrm{~mm}$ as the spacing between wires same as Geometry B as the results produce good convergence among the designs.

\footnotetext{
${ }^{*}$ Corresponding author: nuramirahrashidi@gmail.com
} 


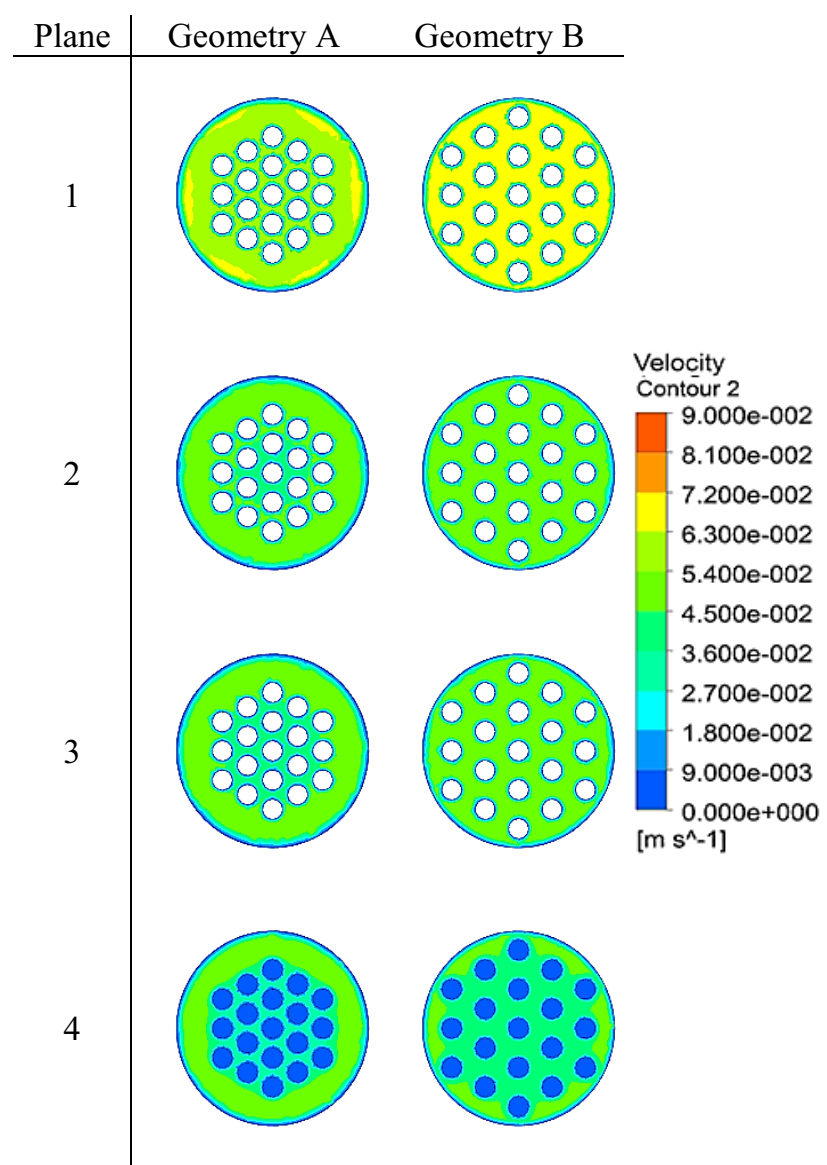

Fig. 5. Velocity contours for $\mathrm{N}_{2}$ flow in $\mathrm{XY}$-axis.

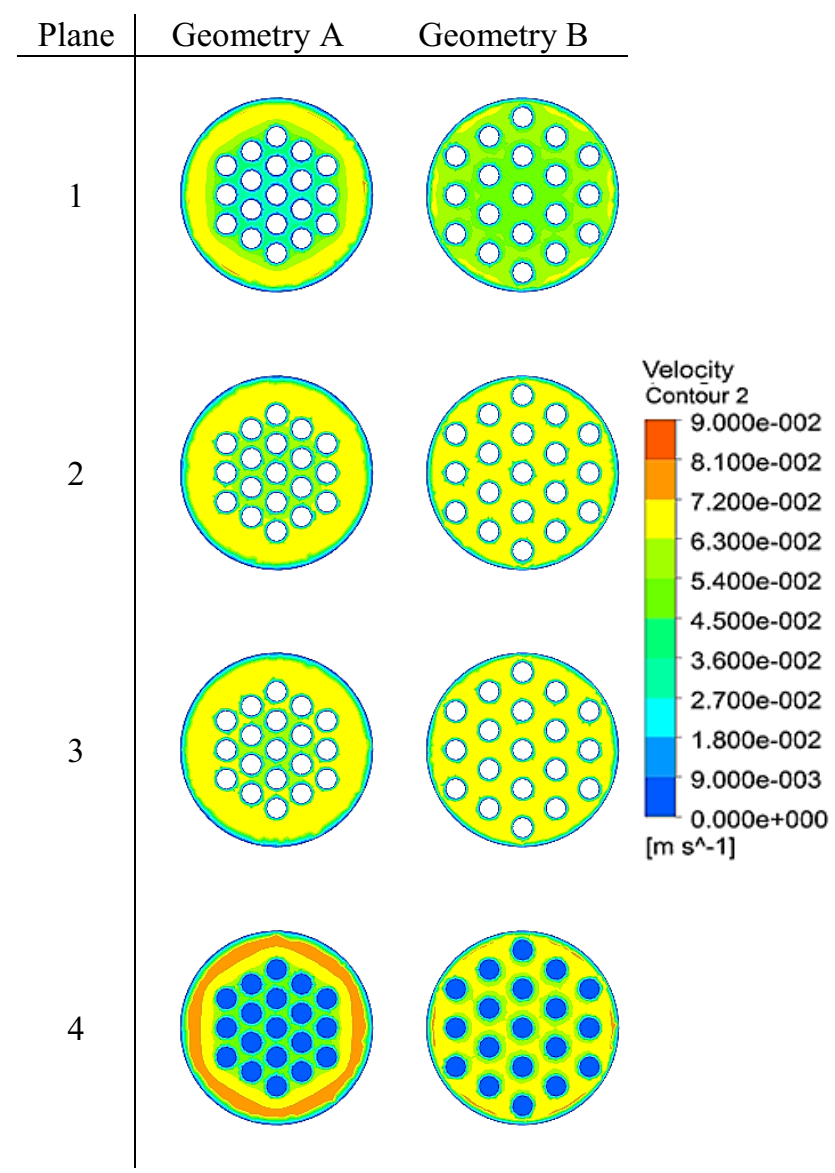

Fig. 6. Velocity contours for $\mathrm{H}_{2}$ flow in $\mathrm{XY}$-axis.

\footnotetext{
${ }^{*}$ Corresponding author: nuramirahrashidi $@$,gmail.com
}

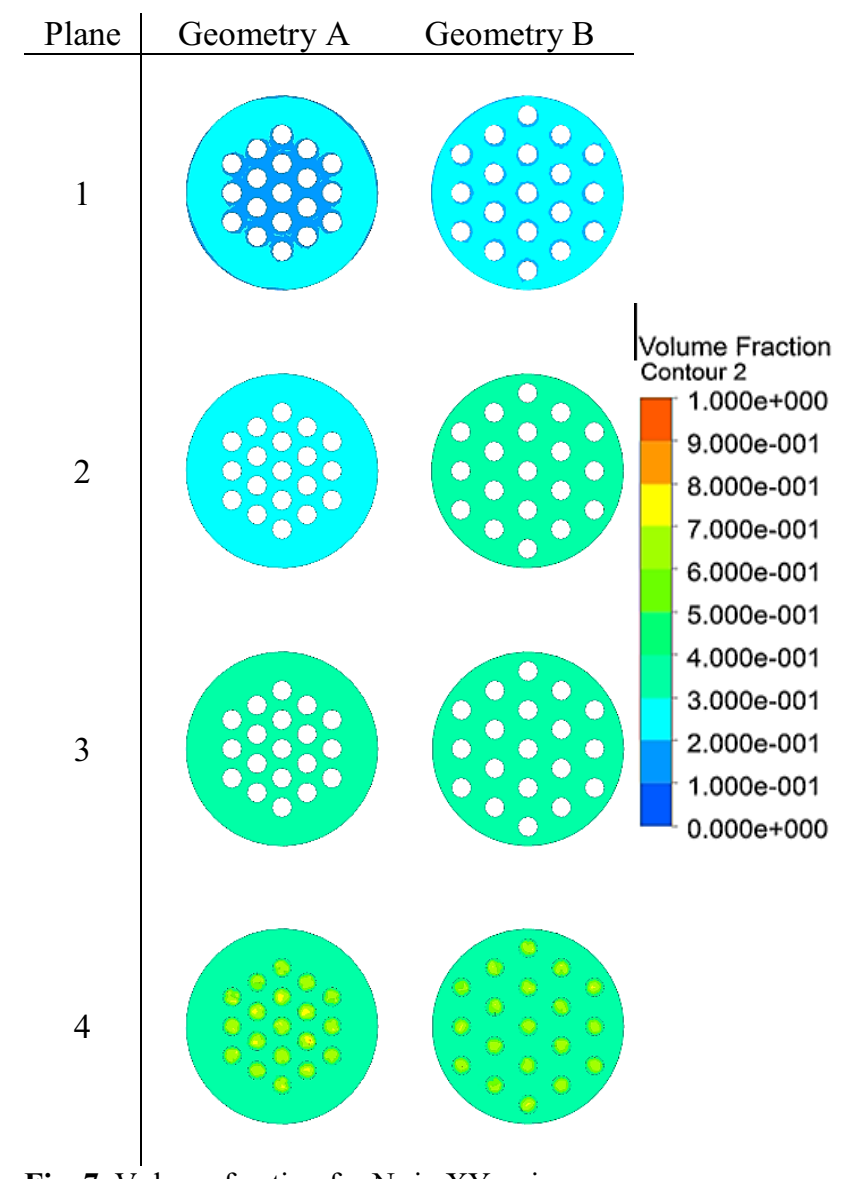

Fig. 7. Volume fraction for $\mathrm{N}_{2}$ in $\mathrm{XY}$-axis.
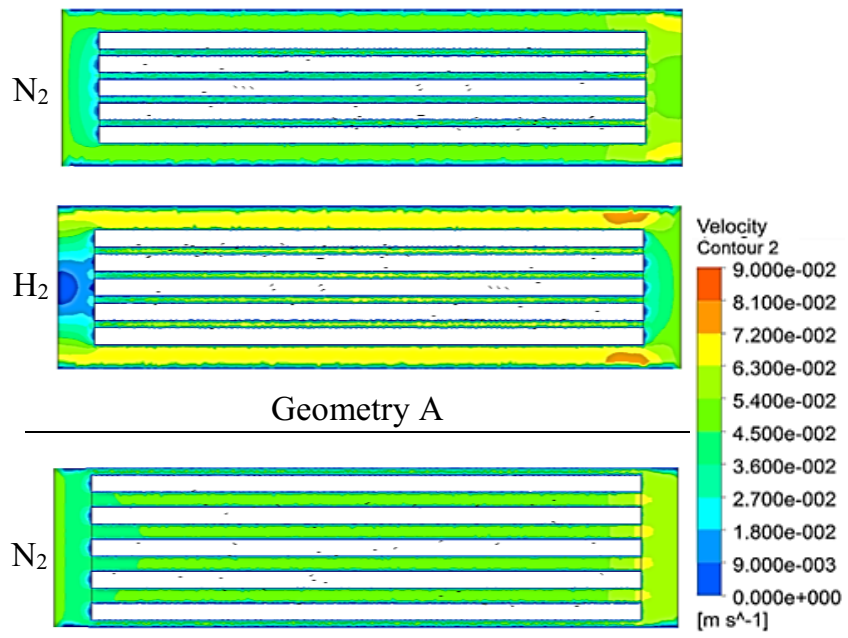

$\mathrm{H}_{2}$

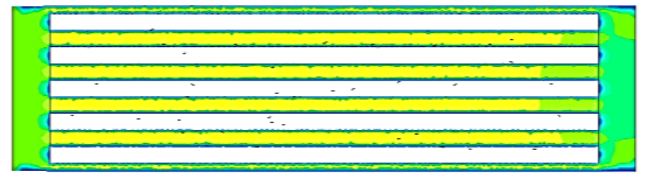

Geometry B

Fig. 8. Velocity contours at centreline in YZ-axis.

\subsection{Effect of numbers of wires}

From the results presented in Figure 9, Geometry C creates more uniform velocity except for plane 1 while Geometry B possess velocity of nitrogen flow approximately at $0.065 \mathrm{~ms}^{-1}$ at the first plane which is 
higher than the set velocity. An active dynamic mixing was expected when the tone of the contour for both gases achieve similarities. Based on the set velocity in Figure 10 , hydrogen gas flows at faster speed for Geometry $\mathrm{C}$ where the velocity is between $0.054 \mathrm{~ms}^{-1}$ and $0.063 \mathrm{~ms}^{-1}$ from plane 1 to 4 while Geometry $B$ retain velocity between $0.063 \mathrm{~ms}^{-1}$ and $0.072 \mathrm{~ms}^{-1}$. Since the velocity of nitrogen and hydrogen for Geometry $\mathrm{C}$ is nearly similar, it will lead to more homogenous mixing.

Plane 1 and 2 in Figure 11 for Geometry $\mathrm{C}$ showed suitable 0.25:0.75 volume fraction ratio while Geometry $\mathrm{B}$ achieve the appropriate volume fraction only in Plane 1. Therefore, lesser or higher volume fraction of the gases will result to lower the yield of ammonia when the actual reaction occurs in the channel.

Velocity of nitrogen and hydrogen can be visualized in Figure 12 at an altered angle where the contour is obtained at the point $\mathrm{Z}=0$ on YZ-axis, showing the radial centerline of the movement for both gases. Similar hue of contour for nitrogen and hydrogen contours along the channel indicating good mixing among the gases. The velocity contours at centreline for Geometry $\mathrm{C}$ illustrated better shifting of gasses when number of wires is lower.

As for the mixing index result, the Figure 13 illustrated that Geometry $\mathrm{C}$ achieved greater dynamic mixing than Geometry B. Hence, we can conclude that lower number of wires, 13 contributes to better mixing efficiency than 19 wires [15] at four different axial distance distributed evenly throughout the monolithic channel.

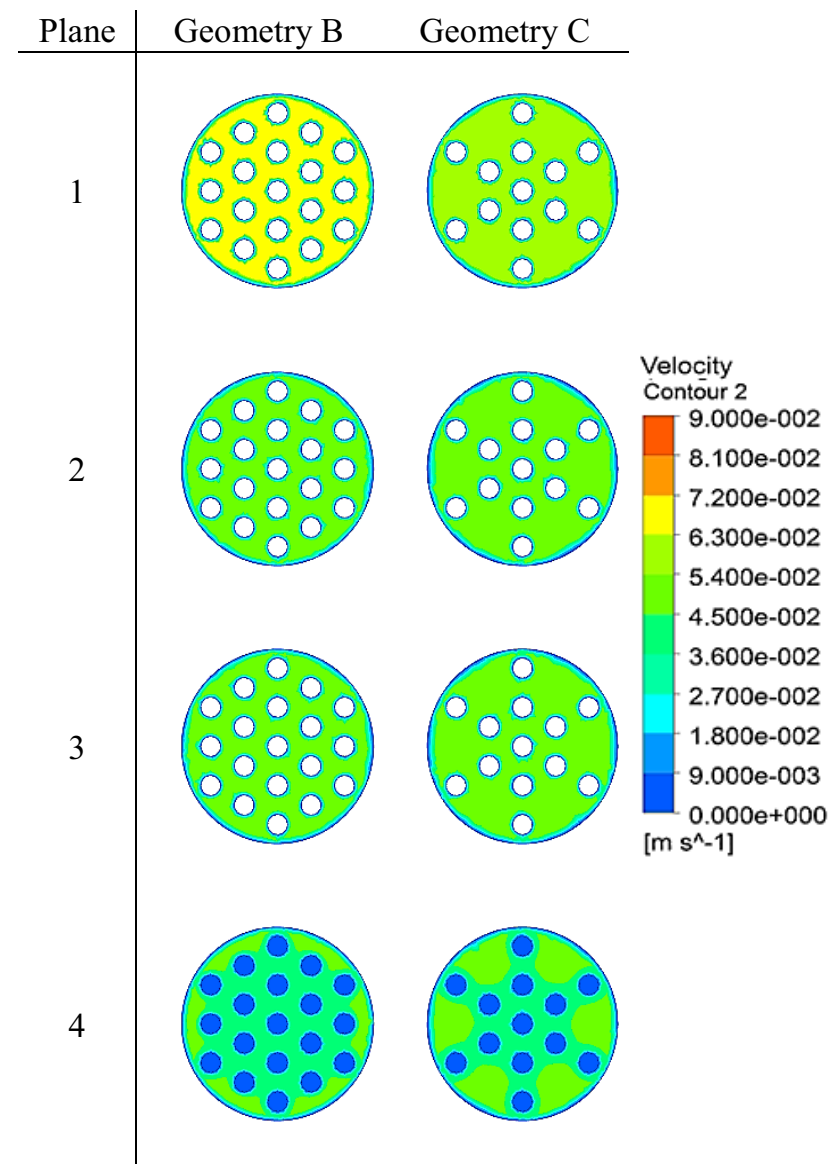

Fig. 9. Velocity contours for $\mathrm{N}_{2}$ flow in $\mathrm{XY}$-axis.

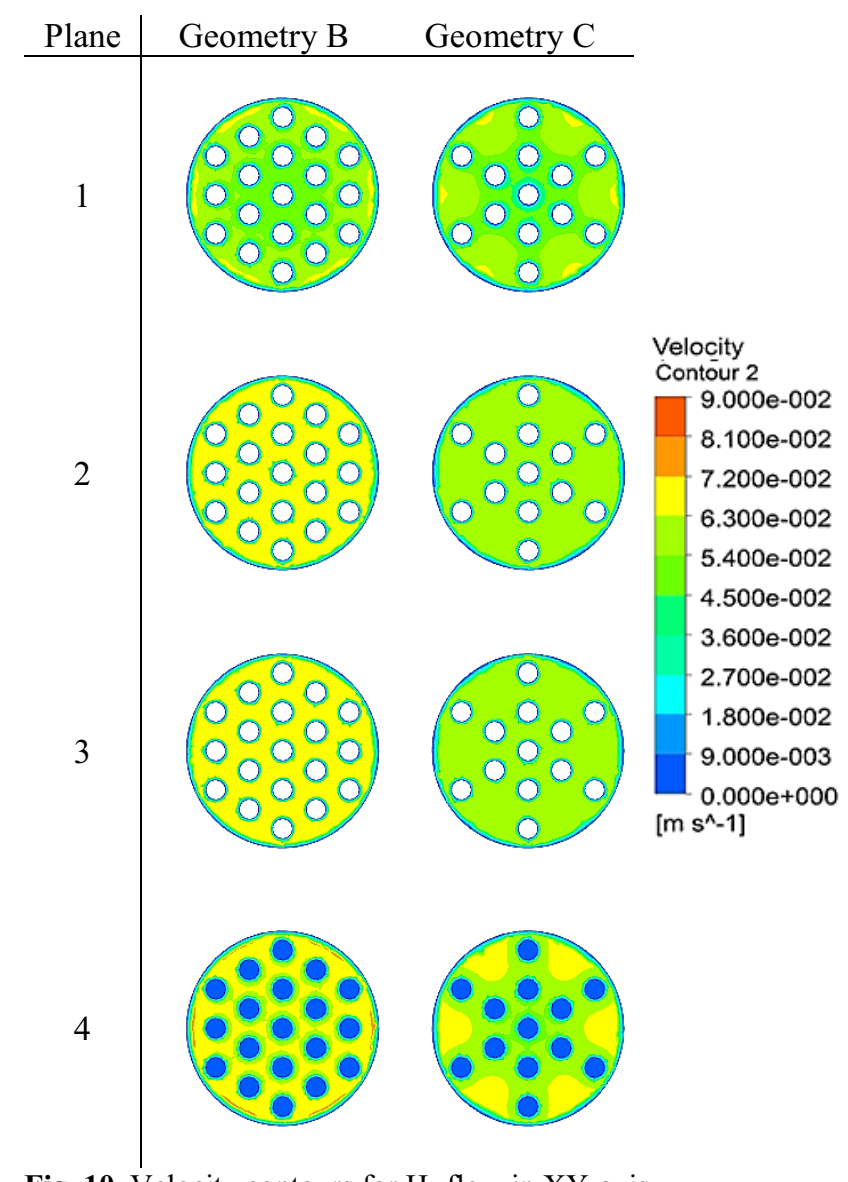

Fig. 10. Velocity contours for $\mathrm{H}_{2}$ flow in $\mathrm{XY}$-axis.

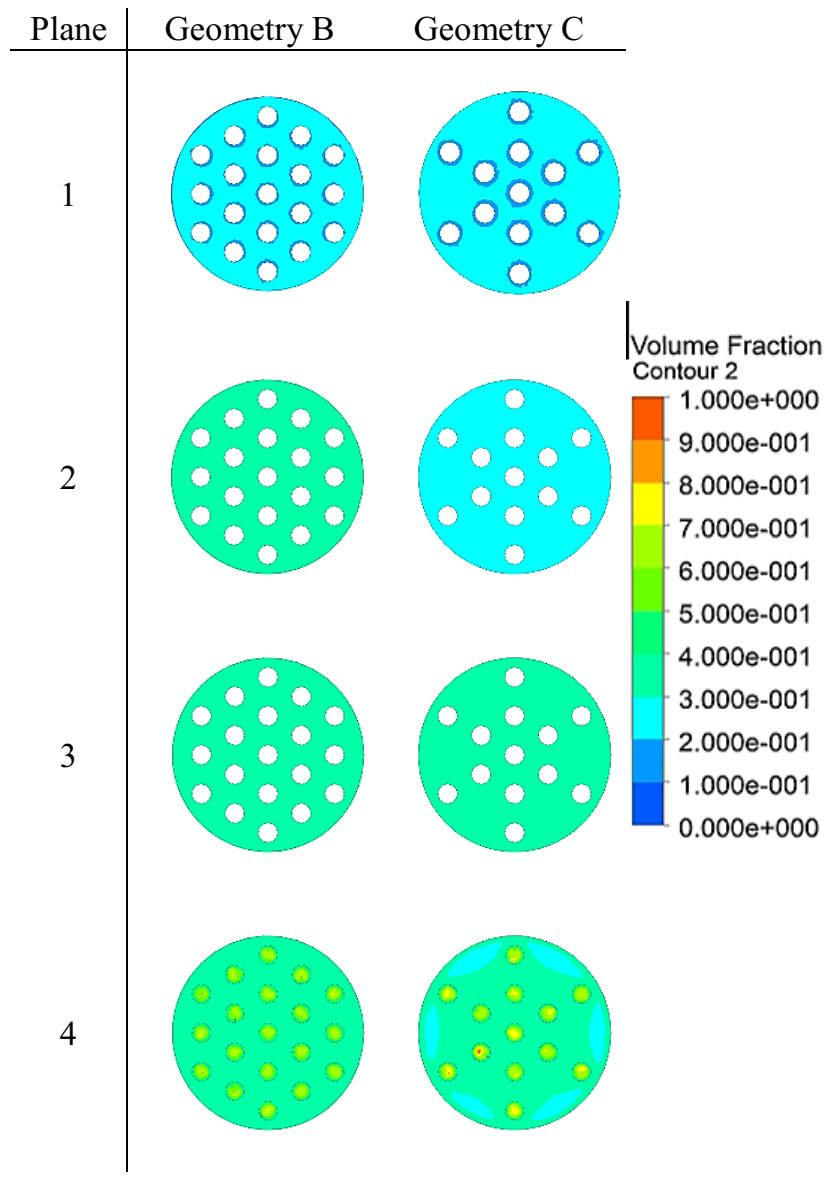

Fig. 11. Volume fraction for $\mathrm{N}_{2}$ in $\mathrm{XY}$-axis.

\footnotetext{
${ }^{*}$ Corresponding author: nuramirahrashidi@gmail.com
} 

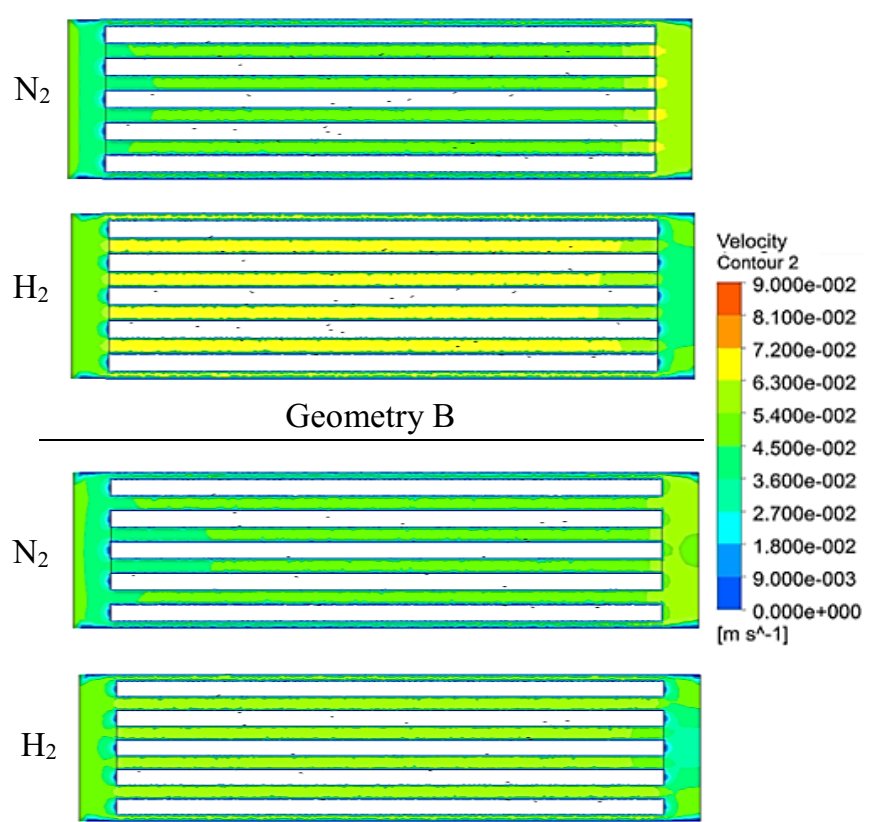

Geometry C

Fig. 12. Velocity contours at centreline in YZ-axis.

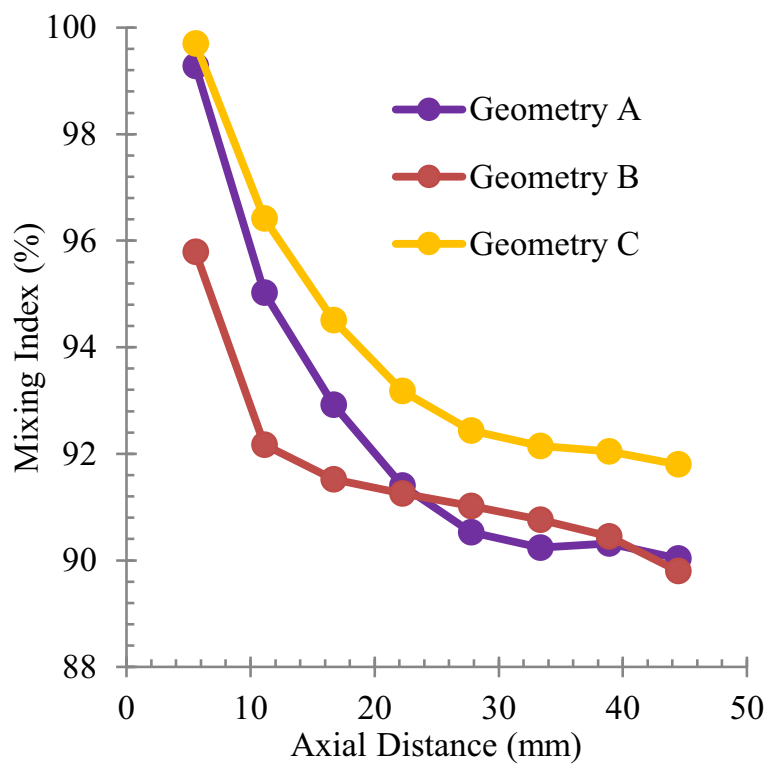

Fig. 13. Mixing index of geometries.

\section{Conclusions}

Enhancing the configuration of wires by increasing spacing and lowering number of wires causing a huge impact on inducing the dynamic mixing of gasses. Geometry $\mathrm{C}$ showed the best mixing result with uniform speed and volume fraction as well as generating high mixing index throughout the monolithic channel. By introducing proper structure of obstructions or wires to the monolithic channel, chaotic advection occurs and the flow direction of the gas stream altered laterally. It is proven that constructing obstruction with larger obstruction space boosted the mixing and rate of reaction between hydrogen and nitrogen gasses to synthesis ammonia. Therefore, the objectives of this project are accomplished successfully with the appropriate methods that have been used. In future, this research will consider the adding of wire supports to hold the wires element in place and inputting magnetic induction into the simulation section to provoke the mixing. Iron based nanocatalyst will be embedded on the wires element configured inside the monolithic channel. The mixing efficiency of the three geometries will be validated using Vibrating Sample Magnetometer (VSM) with various magnetic induction readings from range of 1 to 2.5 Tesla.

The authors gratefully acknowledge Universiti Teknologi PETRONAS for providing CFD computing facilities.

\section{References}

[1] C. Egenhofer, L. Schrefler, The Case of The Chemical Industry - Ammonia (2014)

[2] FAO, Food and Agriculture Organization of the United Nations Rome (2011)

[3] P. Puspitasari, J. A. Razak, N. Yahya, American Institute of Physics (2012)

[4] N. Yahya, P. Puspitasari, K. Koziol, N. A. M. Zabidi, M. F. Othman, International Journal of Basic and Applied Science, 10 60-64 (2010)

[5] K. Salman, A. Zamri, K. Kuzilati, Procedia Engineering, 148 1266-1273 (2016)

[6] W. Wang, S. Zhao, T. Shao, M. Zhang, Y. Jin, Y. Cheng, Chemical Engineering Science, 84 148-154 (2012)

[7] Y. Asano, S. Togashi, H. Tsudome, S. Murakami, International Society for Pharmaceutical Engineering, 30 (2010)

[8] N. Nguyen, Z. Wu, Journal of Micromechanics and Microengineering, 15 (2005)

[9] P. L. Mills, D. J. Quiram, J. F. Ryley, Chemical Engineering Science, $626692-7010$ (2007)

[10] R. B. Liu, M. A. Stremler, K. V. Sharp, M. G. Olsen, J. G. Santiago, R. J. Adrian, H. Aref, D. J. Beebe, Journal of Microelectromechanical Systems, 9 190197 (2000)

[11] R. Wang, J. Lin, H. Li, Chaos, Solitons and Fractals, 33 1362-1366 (2007)

[12]Z. Zhang, C. Yim, M. Lin, X. Cao, Biomicrofluidics, 2 (2008)

[13] C. K. Chung, T. R. Shih, Microfluid Nanofluid, 4 419-425 (2007)

[14] W. W.-F. Leung, Y. Ren, International Journal of Heat and Mass Transfer, 64 457-467 (2013)

[15]H. Shou-Shing, H. Yi-Cheng, Journal of Micromechanics and Microengineering, 18 (2008)

\footnotetext{
${ }^{*}$ Corresponding author: nuramirahrashidi@gmail.com
} 